\title{
Allgemeine Innere Medizin am Universitätsspital
}

\section{Drahomir Aujesky}

Prof. Dr. med., Klinikdirektor und Chefarzt Universitätsklinik und Poliklinik für Allgemeine Innere Medizin, Inselspital Bern

\begin{abstract}
Weil universitäre Medizin mit hochspezialisierter Medizin assoziiert wird, haben Entscheidungsträger oft Mühe, die Bedeutung der Allgemeinen Inneren Medizin am Universitätsspital zu verstehen. Die Betreuung komplexer, multimorbider Patienten wird nicht kostendeckend abgegolten, Kantone reduzieren ihre Weiterbildungsbeiträge teilweise massiv. An Universitätsstandorten braucht es aber einen gesunden Mix zwischen spezialisierter und generalistischer Medizin.
\end{abstract}

\footnotetext{
Die Literaturangaben finden sich unter www.saez.ch $\rightarrow$ Aktuelle Ausgabe oder $\rightarrow$ Archiv $\rightarrow 2015 \rightarrow 3$.
}

\section{Einleitung}

Die universitäre Allgemeine Innere Medizin (AIM) hat durch die fortschreitende Spezialisierung einen Statuswandel erfahren. Früher waren die internistischen Spezialfächer Teil der «Mutterdisziplin» AIM, heute existieren sie als unabhängige Fachdiszipli-

La médecine interne générale dans les hôpitaux universitaires

La médecine interne générale universitaire joue un rôle clé dans la prise en charge des patients polymorbides, la formation globale des étudiants en médecine, la formation postgraduée des futurs généralistes, ainsi que dans la recherche clinique axée sur le patient. Il s'agit d'une discipline intégrative complémentaire aux disciplines spécialisées, dont la fragmentation se poursuit, et qui, en raison de ses liens étroits avec les médecins de famille et la formation des médecins-chefs internistes, joue aussi un rôle déterminant pour les médecins référents et la fidélisation des patients. Etant donné que I'on associe la médecine universitaire à la médecine hautement spécialisée, les décideurs ont souvent des difficultés à comprendre l'utilité de la médecine interne générale au sein d'un hôpital universitaire. Alors que l'indemnisation de la prise en charge des patients polymorbides ne permet pas de couvrir les frais et que certains cantons ont fortement revu à la baisse le financement de la formation postgraduée, la médecine interne générale semble à première vue peu intéressante pour les hôpitaux sur le plan financier. Ces derniers sont donc tentés de réduire leurs investissements dans ce domaine, d'abandonner la médecine de base voire de la reléguer en périphérie, avec pour conséquences une perte d'attractivité et une diminution de la relève qui, au final, impactent également les disciplines spécialisées, les hôpitaux non universitaires et les médecins de famille. Des décideurs inspirés sauront-ils reconnaître le potentiel stratégique de la médecine interne générale et promouvoir une combinaison équilibrée entre médecine spécialisée et médecine générale? nen und Universitätskliniken. Waren es früher die Spezialisten, die sich als Teil der internistischen Klinik über mangelnde Entwicklungsmöglichkeiten beklagt haben, so ist es heute oft die AIM, die eine tiefere Priorität bei den Entscheidungsträgern geniesst. Fehlende Investitionen und Bettenabbau zugunsten der Spezialfächer waren teilweise die Folge, es wurde gar die Berechtigung einer AIM am Universitätsstandort in Frage gestellt. Dieser Beitrag soll die Bedeutung der stationären AIM am Universitätsspital beleuchten.

\section{Klinisches Tätigkeitsfeld}

Die Kernkompetenz der AIM ist die Comprehensive, Coordinated, and Cost-effective Care [1] ${ }^{*}$, im angelsächsischen Raum auch unter dem Begriff Generalism bekannt. Integration verschiedener Diagnosen und Therapien, Setzen von Behandlungsprioritäten sowie Anpassung an die individuellen Bedürfnisse und Möglichkeiten des Patienten sind Kernpunkte des Generalism. Als organübergreifende Disziplin versteht sich die AIM nicht "nur» als Summe der internistischen Spezialdisziplinen, sondern als eigenes Fach mit folgenden Aufgaben im stationären Bereich: 1. Betreuung multimorbider Patienten, 2. Abklärung von unklaren/unspezifischen Symptomen/Befunden und 3. kosteneffektives Management häufiger Pathologien, die primär keiner spezialisierten Behandlung bedürfen. Zudem garantieren die polyvalenten AIMBettenstationen eine rasche Entlastung der Notfall-/ Intensivstation und tragen zu effizienten Patienten- 
flüssen bei. Die AIM übernimmt auch die internistische Versorgung in Spezialfächern und chirurgischen Disziplinen, die sich so besser auf ihre Kernaufgaben konzentrieren können. Zur Erhaltung der internistischen Fachkompetenz sollte dabei eine Anbindung an die AIM bestehen.

Ca. $65 \%$ der über 65-Jährigen sind multimorbid und die Prävalenz multimorbider Personen wird von 2005 bis 2020 um ca. 30\% zunehmen $[2,3]$. Multimorbidität ist assoziiert mit tieferer Lebenserwartung, reduziertem funktionellem Status, sozialen Problemen und höheren Gesundheitskosten $[2,3]$. Da bei Multimorbidität nicht unbedingt die Krankheit eines Einzelorgans im Vordergrund steht, braucht es für eine optimale Behandlungsqualität und -effizienz ein integratives Fach wie die AIM, das einerseits häufige Probleme abschliessend managt, andererseits komplexe Behandlungsprozesse zusammen mit Spezialisten und Hausärzten koordiniert und auf den Patienten abstimmt. Die unkoordinierte Betreuung durch multiple Ärzte würde zur Fragmentierung führen, verbunden mit Qualitätsverlust und Kostenzunahme [4].

Zwangsläufig besteht mit den aus der Inneren Medizin hervorgegangenen Spezialfächern eine Überschneidung im Leistungsangebot, was spitalintern zu Konkurrenz führen kann. Dabei lässt sich der Anspruch einer generell besseren Behandlungsqualität durch Spezialisten nicht belegen [4-6]. Patienten mit kom-

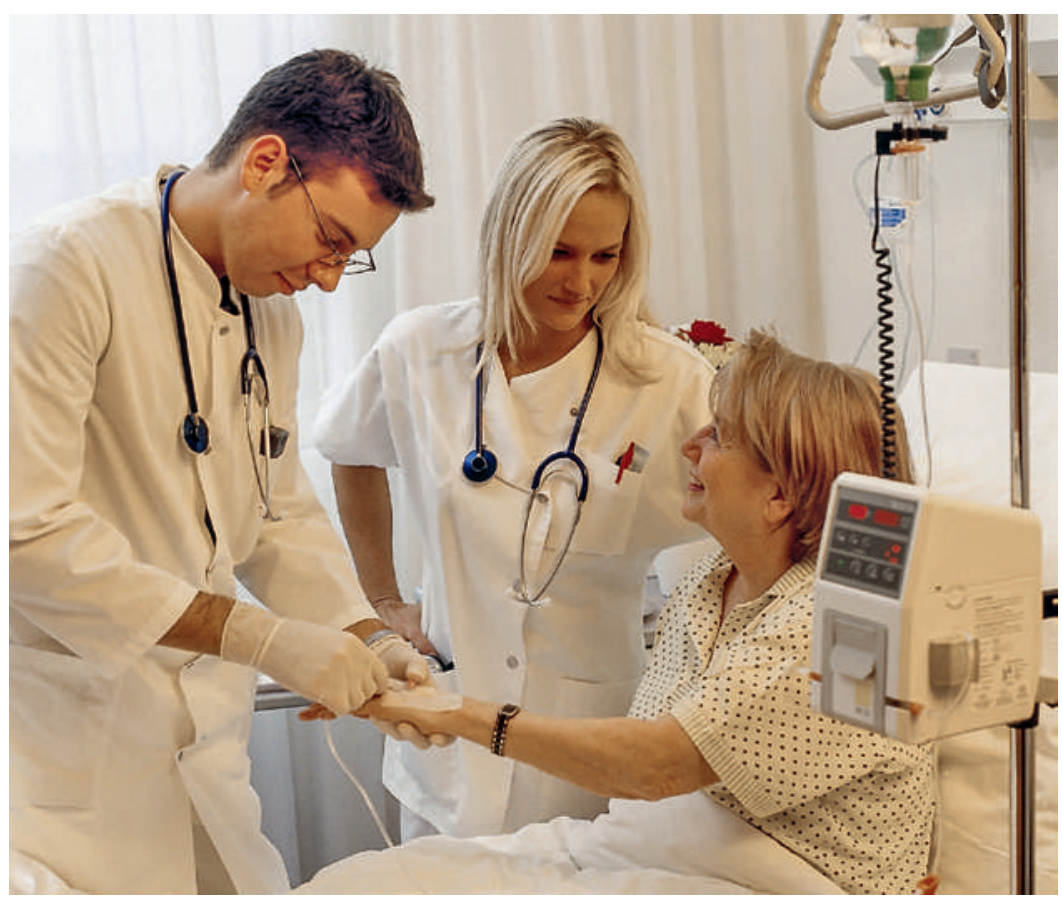

Das breite Weiterbildungsangebot und das Prestige, an einem Universitätsstandort zu arbeiten, machen die Weiterbildung an einer universitären Abteilung für Allgemeine Innere Medizin attraktiv. plexen (Einzel-)Erkrankungen und komplexen Therapien (z.B. Interventionen) gehören primär in die Hand des Spezialisten $[1,7]$. Komplexe Patienten mit Mehrfacherkrankungen und solche mit alltäglicheren Problemen, wo für das Management durch Spezialisten

\section{Zwangsläufig besteht mit den aus der Inneren} Medizin hervorgegangenen Spezialfächern eine Überschneidung im Leistungsangebot.

kein besseres Kosten-/Nutzen-Verhältnis zu erwarten ist, sollten primär von Generalisten betreut werden, mit selektivem Beizug von Spezialisten $[1,7]$. Spezialisten und Generalisten sind komplementär und erzielen in Zusammenarbeit die besten Resultate [8].

\section{Weiterbildung und Kaderausbildung}

Da die Weiterbildung zum Facharzt für Allgemeine Innere Medizin aus minimal 2 Jahren stationärer AIM besteht, hat diese einen wesentlichen Anteil an der Weiterbildung zum Grundversorger. Der universitären AIM kommt aufgrund ihrer Grösse eine besondere Rolle zu: Von den ca. 9o Assistenz- und Oberärzten der Universitätsklinik für Allgemeine Innere $\mathrm{Me}$ dizin am Inselspital gehen ca. 60\% in die Grundversorgung. Das breite Weiterbildungsangebot, einschliesslich Rotationen in Spezialfächern, und das Prestige, an einem Universitätsstandort zu arbeiten, machen die Weiterbildung an einer universitären AIM attraktiv. Die aufgrund der Klinikgrösse tiefere Dienstbelastung und Möglichkeit zur Teilzeitarbeit kommen gesellschaftlichen Trends entgegen. Da die Assistenzärzte auf der AIM pro Zeiteinheit mit vielen Diagnosen in Kontakt kommen und sich am Universitätsspital auch seltenere Krankheitsbilder konzentrieren, ist die Weiterbildung äusserst breit und effizient. Um eine optimale Weiterbildung anbieten zu können, müssen drei Voraussetzungen gegeben sein: 1. Zugang zu einem breiten Patientengut, 2. genügend Rotationen in Notfall- und Intensivmedizin und 3. ein ausgewogenes Verhältnis zwischen Weiterbildung im Kernbereich der AIM und Rotationen in Spezialfächern, da bei zu fragmentierter Weiterbildung keine optimale Integration und Synthese stattfinden kann. Ziel ist es, eine genügende Anzahl von marktfähigen Generalisten weiterzubilden, die das wachsende Bedürfnis an Grund- und Spitalversorgung abdecken. Zudem dienen die Ärzte der AIM als gut ausgebildeter Rekrutierungspool für Spezialfächer. Um Assistenzärzte auf ihre Tätigkeiten in Spital und Praxis vorzubereiten, müssen AIM-Univer- 
sitätskliniken als Weiterbildungs-Hubs zusammen mit Netzwerk-Spitälern, Hausarzt-Instituten und Praxispartnern personalisierte Weiterbildungscurricula anbieten, einschliesslich spezifischer HausarztTracks. Eine weitere Aufgabe der universitären AIM ist es, Nachwuchstalente auf eine Chef- und Kaderfunktion hin $\mathrm{zu}$ fördern. Neben der klinischen

\section{Die AIM hat für das Universitätsspital eine wachsende strategische Bedeutung in Bezug auf Patienten- und Zuweiserakquisition und -anbindung.}

Weiterbildung gehört dazu die Möglichkeit zur akademischen Entwicklung und Leadership-/Management-Erfahrung.

Obwohl von Angebotsstrategen oft nicht erkannt, hat die AIM für das Universitätsspital eine wachsende strategische Bedeutung in Bezug auf Patienten- und Zuweiserakquisition und -anbindung. Die stationäre Behandlung multimorbider Patienten ist zwar in vielen Fällen nicht rentabel, führt aber durch längerfristige Patientenanbindung zum Bezug von besser abgegoltenen Leistungen. Oft rekrutieren sich die regionalen Grundversorger aus der universitären AIM oder unterstützen als Generalisten deren ganzheitlichen Approach. Daraus kann eine langfristige Zusammenarbeit mit dem Universitätsspital entstehen, die über persönliche Kontakte und Fortbildungen zementiert wird. Die Besetzung von internistischen Chefarztstellen an nicht-universitären Spitälern durch Kaderärzte des universitären «Mutterhauses» ist für strategische Kooperationen von kapitaler Bedeutung.

\section{Lehre und Forschung}

Zusammen mit den Hausarztinstituten ist die universitäre AIM eines der letzten Fächer, das Medizinstudenten einen ganzheitlichen Approach vermittelt und zur Berufswahl des Generalisten motiviert [9]. Die AIM-Forschung bewegt sich weg von der traditionellen Grundlagen- und translationalen Forschung ("from bench to bedside») zur patientenzentrierten klinischen Forschung, deren Ziel die direkte Verbesserung der Behandlungsqualität ist. Zentral für die AIM sind die Beforschung multimorbider, polymedikalisierter Patienten unter Real-life-Bedingungen und die Berücksichtigung von patientenrelevanten Outcomes, wie funktionaler Status, Symptomlast und aktive Lebenserwartung. Die patientenzentrierte Forschung ist komplementär zur
Grundlagenforschung, deren Resultate nur nach Jahren klinisch anwendbar sind [10].

\section{Problemzonen}

Universitäre Medizin wird zu Unrecht oft mit hochspezialisierter Medizin gleichgesetzt. Da die AIM mehr auf Prozesse wie Integration und nicht auf ein/e konkrete/s Organsystem/Krankheit fokussiert, haben Entscheidungsträger oft Mühe, die Rationale des "Gemischtwarenladens» AIM am Universitätsspital $\mathrm{zu}$ verstehen [11]. Zudem konzentrieren sich auf der AIM multimorbide Patienten mit langen Aufenthaltsdauern (oft zur Entlastung anderer Fächer!), die unter SwissDRG trotz effizienten Prozessen nicht kostendeckend abgegolten werden. Kantone haben ihre Weiterbildungsbeiträge teilweise massiv reduziert, worunter die universitäre AIM mit ihren hohen Assistentenzahlen besonders leidet. Die Finanzschwäche der AIM ist ihre Achillesferse, da sie anders als finanzkräftige Spezialfächer Entscheidungsträger weniger beeinflussen kann [12]. Mögliche Folgen sind die Benachteiligung der universitären AIM in Bezug auf Investitionen und Abbau oder Auslagerung der «Grundversorgung» an nicht-universitäre Standorte, mit Verlust an Sichtbarkeit/Prestige des Fachs. Die Konsequenzen sind Attraktivitätsverlust, Entakademisierung und ein Nachwuchsmangel, den

Die Frage ist, ob visionäre Verwaltungsräte und Geschäftsleitungen das Potential der AIM erkennen.

auch die internistischen Spezialfächer, nicht-universitäre Spitäler und Grundversorger zu spüren bekommen. Weiter droht die Fragmentierung der Betreuung multimorbider Patienten, Entfremdung zwischen Universitätsspital und Grundversorgern und eine Verschiebung im regionalen Ärztemix hin zur Spezialmedizin.

Auf Fakultätsebene bleibt die traditionelle Grundlagenforschung der Goldstandard [11], patientenzentrierte klinische Forschung wird oft als weniger prestigeträchtig empfunden. Wegen der im Vergleich mit der Spezialmedizin geringeren Anzahl akademischer Generalisten findet in der studentischen Lehre teilweise eine Verschiebung vom ganzheitlichen Ansatz zu relativ eng umschriebenen Themen der spezialisierten Medizin statt [13, 14], mit möglicher Schwächung des Generalisten als Rollenmodell und Verschiebung der Berufswahl weg vom Generalism [15]. 


\section{Beispiele von Fehlentwicklungen}

1999 erfolgte am Inselspital Bern die Auflösung des Departements für Innere Medizin mit Reduktion der AIM auf eine kleine Bettenstation [16]. Nur ein Jahr später musste die AIM wegen suboptimaler Prozessabläufe bei multimorbiden Patienten und Weiterbildung auf Druck der Ärztegesellschaft wieder auf über 110 Betten aufgebaut werden.

An deutschen Universitätskrankenhäusern ist die AIM als akademisches Fach im Zuge der Spezialisierung fast verschwunden, AIM-Abteilungen existieren fast nur noch an peripheren Krankenhäusern [17]. Multimorbide Patienten werden in organ-, krankheitsoder alterszentrierten Schwerpunktkliniken behandelt. Ärzte in Weiterbildung zum Internisten oder Allgemeinmediziner rotieren durch diese Kliniken und versuchen, sich möglichst viel Fachwissen anzueignen. Die Weiterbildung ist fragmentiert [18], eine über den Bereich des Schwerpunkts hinausgehende Synthese oder ganzheitliche Beurteilungsweise kann nur schwer erlernt werden. Die deutschen Kollegen schätzen die strukturierte, ganzheitliche Weiterbildung an Schweizer AIM-Universitätskliniken und bewerben sich häufig. Infolge der fragmentierten Versorgung, heterogenen Weiterbildungsqualität und Abwanderung des Nachwuchses werden in Deutschland Forderungen nach dem Wiederaufbau einer integrierten Inneren Medizin und restriktiveren Kriterien zur Weiterbildungsbefugnis in Innerer Medizin laut [19, 20].

In den USA hat die infolge der Spezialisierung fast verschwundene akademische AIM eine eindrückliche Wiedergeburt erlebt. Vor 1970 hatten gerade 5\% der U.S.-Universitätsspitäler eine Abteilung für AIM, 2002 wieder fast alle [21]. Gründe für den Wiederaufbau waren die Krise der Grundversorgung und die Wahrnehmung, dass das U.S.-Gesundheitssystem zu spezialistenlastig und zu teuer sei [22]. Die Universitätsspitäler wurden kritisiert, sie seien nicht fähig oder willens, den richtigen Ärztemix zu produzieren
[23]. Treibende Kräfte für die Renaissance der akademischen AIM waren staatlich finanzierte Weiterbildungsprogramme für Grundversorger sowie die Erkenntnis um die Wichtigkeit einer koordinierten, umfassenden Patientenbetreuung [21, 22]. Heute ist die universitäre AIM in den USA ein Motor der Innovation in Lehre, Weiterbildung, klinischer Epidemiologie und Versorgungsforschung [24].

\section{Ausblick}

Bei rascher Zunahme multimorbider Patienten und steigendem Bedarf an Generalisten (bis 2030 können 40\% des ambulanten Konsultationsvolumens nicht mehr abgedeckt werden [25]) müsste die Zukunft der universitären AIM gesichert sein. Diese wird letztlich aber durch Strukturentscheide an den Universitätsspitälern bestimmt [19]. Die Frage ist, ob visionäre Verwaltungsräte und Geschäftsleitungen das Potential der AIM erkennen und am Universitätsspital einen gesunden Mix zwischen spezialisierter und generalistischer Medizin fördern. Setzt der Universitätsstandort zu einseitig auf Spezialmedizin, drohen Fehlentwicklungen wie in Deutschland. Da ein höherer Grundversorgeranteil zu einer qualitativ besseren und kosteneffizienteren Gesundheitsversorgung führt [26], sind auch die Kantone gefordert, durch eine Erhöhung ihrer Weiterbildungsbeiträge für Generalistenfächer zum Erhalt der Grundversorgung beizutragen. Um die Zukunft der AIM finanziell zu sichern, muss die Verbesserung der Abgeltung von multimorbiden Spitalpatienten eine vordringliche Aufgabe der betroffenen Fachgesellschaften und der Spitäler sein. Schliesslich braucht es einen Schulterschluss der ambulant und stationär tätigen Generalisten, deren Existenz als Berufsgruppe eng miteinander verbunden ist.

\section{Danksagung}

Mein Dank gilt Prof. Dr. med. Nicolas Rodondi für die kritische Durchsicht des Manuskripts. 


\section{Literatur}

1 Larson EB, Fihn SD, Kirk LM, et al. The future of general internal medicine. Report and recommendations from the Society of General Internal Medicine (SGIM) Task Force on the Domain of General Internal Medicine. J Gen Intern Med. 2004;19:69-77.

2 Barnett K, Mercer SW, Norbury M, Watt G, Wyke S, Guthrie B. Epidemiology of multimorbidity and implications for health care, research, and medical education: a cross-sectional study. Lancet. 2012;380:37-43.

3 Vogeli C, Shields AE, Lee TA, et al. Multiple chronic conditions: prevalence, health consequences, and implications for quality, care management, and costs. J Gen Intern Med. 2007;22 Suppl 3:391-5.

4 Detsky AS, Gauthier SR, Fuchs VR. Specialization in medicine: how much is appropriate? JAMA. 2012;307:463-4. 5 Donohoe MT. Comparing generalist and specialty care: discrepancies, deficiencies, and excesses. Arch Intern Med. 1998;158:1596-6o8.

6 Smetana GW, Landon BE, Bindman AB, et al. A comparison of outcomes resulting from generalist vs specialist care for a single discrete medical condition: a systematic review and methodologic critique. Arch Intern Med. 2007;167:10-20. 7 Tinetti ME, Fried TR, Boyd CM. Designing health care for the most common chronic condition - multimorbidity. JAMA. 2012;307:2493-4

8 Ayanian JZ, Landrum MB, Guadagnoli E, Gaccione P. Specialty of ambulatory care physicians and mortality among elderly patients after myocardial infarction. N Engl J Med. 2002;347:1678-86.

9 Bland CJ, Meurer LN, Maldonado G. Determinants of primary care specialty choice: a non-statistical meta-analysis of the literature. Acad Med. 1995;70:620-41.

10 Rosenberg RN. Translating biomedical research to the bedside: a national crisis and a call to action. JAMA. 2003;289:1305-6.

11 Armstrong K, Keating NL, Landry M, et al. Academic general internal medicine: a mission for the future. J Gen Intern Med. 2013;28:845-51.

12 Friedman RH, Alpert JJ, Green LA. Strengthening academic generalist departments and divisions. J Gen Intern Med. 1994;9:S90-8.

13 Noble J. General internal medicine in internal medicine: at the core or on the periphery? Ann Intern Med. 1992;116:1058-60.
14 Rich EC, Wilson M, Midtling J, Showstack J. Preparing generalist physicians: the organizational and policy context. J Gen Intern Med. 1994;9:S115-22.

15 Campos-Outcalt D, Senf J, Watkins AJ, Bastacky S. The effects of medical school curricula, faculty role models, and biomedical research support on choice of generalist physician careers: a review and quality assessment of the literature. Acad Med. 1995;70:611-9.

16 Leu F. Das Inselspital: Geschichte des Universitätsspitals Bern, 1954-2004. Weber, Thun/Gwatt 2006. 2005.

17 Lehnert H. Structure of inpatient range of services of internal medicine and its priorities in Germany. Internal medicine survey of the "structure-hospital care» committee of the German Society of Internal Medicine. Med Klin (Munich). 2009;104:171-6.

18 Schmidt M. About the Fiasco of Vocational Training in General Practice - a Personal Appraisal from a Trainee. Z Allg Med. 2008;84:10-3.

19 Kolloch R. Evolution of medicine as a continuing challenge: opening address of the 115th annual meeting of the German Society of Internal Medicine 19 April 2009 in Kurhaus Wiesbaden]. Med Klin (Munich). 2009;104:837-40.

20 Hallek M, Schroter T. The future of clinical training in internal medicine in Germany. Dtsch Med Wochenschr. 2012;137:2591-4.

21 Levinson W, Linzer M. What is an academic general internist? Career options and training pathways. JAMA. 2002;288:2045-8.

22 Centor RM, Huddle TS. Academic general internal medicine: past, present, future. Am J Med. 2006;119:172-5.

23 Rogers DE, Blendon RJ. The academic medical center: a stressed American institution. N Engl J Med. 1978;298: 940-50.

24 Kravitz RL, Feldman MD. General internal medicine as an engine of innovation. J Gen Intern Med. 2013;28:749-50.

25 Seematter-Bagnoud L, Junod J, Jaccard-Ruedin H, Roth M, Foletti C, Santos-Eggimann B. Angebot und Inanspruchnahme ambulanter medizinischer Dienstleistungen in der Schweiz - Prognosen bis 2030. Arbeitsdokument 33. Neuchâtel. Schweizerisches Gesundheitsobservatorium. 2008.

26 Starfield B, Shi L, Macinko J. Contribution of primary care to health systems and health. Milbank Q. 2005;83:457-502. 